OPEN ACCESS

Edited by:

Paul D. Brown,

University of the West Indies, Jamaica

Reviewed by:

Atte Von Wright,

University of Eastern Finland, Finland John Elmerdahl Olsen,

University of Copenhagen, Denmark

*Correspondence:

Mrutyunjay Suar

msuar@kiitbiotech.ac.in

Specialty section:

This article was submitted to

Infectious Diseases,

a section of the journal

Frontiers in Microbiology

Received: 04 February 2016

Accepted: 11 April 2016

Published: 26 April 2016

Citation:

Ryan D, Ojha UK, Jaiswal S, PadhiC and SuarM (2016) The Small RNA Dsra Influences the Acid Tolerance Response and Virulence of Salmonella enterica Serovar Typhimurium. Front. Microbiol. 7:599. doi: 10.3389/fmicb.2016.00599

\section{The Small RNA DsrA Influences the Acid Tolerance Response and Virulence of Salmonella enterica Serovar Typhimurium}

\author{
Daniel Ryan, Urmesh K. Ojha, Sangeeta Jaiswal, Chandrashekhar Padhi and \\ Mrutyunjay Suar*
}

Infection Biology Laboratory, School of Biotechnology, KIIT University, Bhubaneswar, India

The Gram-negative, enteropathogen Salmonella enterica serovar Typhimurium ( $S$. Typhimurium) is exposed to various stress conditions during pathogenesis, of which acid stress serves as a major defense mechanism in the host. Such environments are encountered in the stomach and Salmonella containing vacuole of phagocytic and nonphagocytic cells. It is only recently that small RNAs (sRNAs) have come to the forefront as major regulators of stress response networks. Consequently, the sRNA DsrA which regulates acid resistance in Escherichia coli, has not been characterized in the acid tolerance response (ATR) of Salmonella. In this study, we show dsrA to be induced two and threefold under adaptation and challenge phases of the ATR, respectively. Additionally, an isogenic mutant lacking $d s r A(\triangle D s r A)$ displayed lower viability under the ATR along with reduced motility, feeble adhesion and defective invasion efficacy in vitro. Expression analysis revealed down regulation of several Salmonella pathogenicity island-1 (SPI-1) effectors in $\triangle \mathrm{DsrA}$ compared to the wild-type, under SPI-1 inducing conditions. Additionally, our in vivo data revealed $\triangle \mathrm{DsrA}$ to be unable to cause gut inflammation in C57BL/6 mice at $72 \mathrm{~h}$ post infection, although intracellular survival and systemic dissemination remained unaffected. A possible explanation may be the significantly reduced expression of flagellin structural genes fliC and fljB in $\triangle \mathrm{Dsr} A$, which have been implicated as major proinflammatory determinants. This study serves to highlight the role of sRNAs such as DsrA in both acid tolerance and virulence of $S$. Typhimurium. Additionally the robust phenotype of non-invasiveness could be exploited in developing SPI-I attenuated S. Typhimurium strains without disrupting SPI-I genes.

Keywords: sRNA, ATR, virulence, inflammation, mice, adhesion, invasion, motility

\section{INTRODUCTION}

Salmonella enterica are a group of Gram-negative bacteria capable of causing clinical symptoms ranging from diarrhea to severe enteritis as well as life threatening systemic infections (Galán, 2001). During pathogenesis, Salmonella are exposed to an array of hostile conditions such as acidic pH, reactive oxygen species, bile salts, etc (Suar and Ryan, 2015). One such stress most frequently encountered is low $\mathrm{pH}$ and the ability of Salmonella to sense and respond to this is essential to its survival strategy. Salmonellae are exposed to acidic $\mathrm{pH}$ primarily in the stomach 
and within the Salmonella containing vacuole of phagocytic and non-phagocytic cells (Allam et al., 2012). This acidification serves as a major defense of the host and promotes the killing effects of other bactericidal mechanisms namely increased formation of phagolysosomes, activation of acid hydrolases and formation of free radical species. Following acid exposure, Salmonellae adopt different survival strategies collectively termed the acid tolerance response (ATR; Foster and Hall, 1990). Under this response, when $S$. enterica subspecies enterica Serovar Typhimurium ( $S$. Typhimurium) cells are grown at sub-lethal $\mathrm{pH}(4.5-5.5)$ for one generation, they gain the ability to survive at normally lethal $\mathrm{pH}$ levels (Baik et al., 1996). During this adaptation, the cell synthesizes a number of acid shock proteins (ASPs) at moderate $\mathrm{pH}$ levels that provide protection on subsequent exposure to severe acid (Foster, 1993).

To date, a number of studies have characterized the roles of various protein coding genes and regulators associated with the ATR (Foster, 1993; Audia et al., 2001). However, there has been very little emphasis on the small RNA (sRNA) complement associated with such a response. It is of late that sRNAs have come to the forefront as master regulators within a cell and have been shown to be highly involved in stress response and virulence (Gong et al., 2011; Hébrard et al., 2012). They are generally 50-500 nucleotides in length, ubiquitous in all kingdoms of life and normally do not code for protein products (Ryan et al., 2015). They function by base pairing to target mRNAs and bring about either repression or activation by affecting mRNA stability or exposing the ribosome binding site, respectively. Binding may sometimes involve the RNA chaperone Hfq to facilitate the sRNA-mRNA interaction. Several sRNAs may also bind directly to proteins and modify their functions (Altuvia, 2007).

The 87 nucleotide sRNA DsrA present in both Escherichia coli (E. coli) and Salmonella has been shown to be a regulator of acid resistance in the former only (Lease et al., 2004). Additionally, DsrA has also been shown to be repressed in non-proliferating $S$. Typhimurium within fibroblasts suggesting a possible role in the extracellular environment (Ortega et al., 2012). Two targets regulated by DsrA in E. coli include the stress sigma factor rpoS and the histone-like nucleoid protein $h n s$, both of which regulate genes involved in stress response and virulence (Nadim et al., 1998). Furthermore, E. coli $d s r A$ mutants were found to be acid sensitive (Lease et al., 2004). To this effect, the present work was undertaken to characterize the role of DsrA in the ATR and virulence of the enteropathogen, $S$. Typhimurium.

\section{MATERIALS AND METHODS}

\section{Bacterial Strains and Growth Conditions}

The strains and plasmids used in this study are listed in Table 1. Streptomycin (Sm; $50 \mu \mathrm{g} / \mathrm{ml}$ ), kanamycin (Km; $50 \mu \mathrm{g} / \mathrm{ml}$ ), chloramphenicol (Cm; $20 \mu \mathrm{g} / \mathrm{ml}$ ) and ampicillin (Amp; $100 \mu \mathrm{g} / \mathrm{ml}$ ) were added as appropriate to selected strains. Growth kinetics and ATR assays were performed in minimal $E$ glucose medium (MEM; Vogel and Bonner, 1956). The medium was supplemented with dextrose (sterilized with $0.22 \mu \mathrm{m}$ filter) at a concentration of $0.4 \%$ and casein hydrolysate (sterilized with $0.22 \mu \mathrm{m}$ filter) at a concentration of $0.1 \%$ to allow for $S$. Typhimurium SB300 growth.

Growth kinetics in MEM were determined by growing strains in triplicate over a period of $8 \mathrm{~h}$ at $37^{\circ} \mathrm{C}$ and $180 \mathrm{rpm}$. For the initial $2 \mathrm{~h}$ post subculture, $\mathrm{OD}_{600}$ measurements and plating of appropriate dilutions was done every $30 \mathrm{~min}$. Subsequently, $\mathrm{OD}_{600}$ measurements and plating were performed every hour up to a total of $8 \mathrm{~h}$.

Log-phase ATR was induced as described previously. (Baik et al., 1996). Briefly, a single colony of overnight grown $S$. Typhimurium SB300 culture in $5 \mathrm{ml} \mathrm{MEM}\left(37^{\circ} \mathrm{C}\right.$ and $\left.180 \mathrm{rpm}\right)$ was sub-cultured (1:100 dilution) into $100 \mathrm{ml}$ flasks and incubated until an $\mathrm{OD}_{600}$ of 0.4 was attained. Following this, cultures to be adapted were adjusted to $\mathrm{pH} 4.4( \pm 0.1)$ with $3 \mathrm{~N}$ $\mathrm{HCl}$ and incubated for $1 \mathrm{~h}$. Adapted cultures were subsequently challenged at $\mathrm{pH} 3.1( \pm 0.1)$ as above and incubated for 1,2 , and $4 \mathrm{~h}$ post challenge. Colony forming units (CFUs) were calculated after plating appropriate dilutions on agar plates. Percent viability was calculated by dividing the CFU at different time points post challenge by CFU prior to challenge and multiplying the result by 100. ATR induction experiments were performed in triplicate.

\section{Construction of dsrA Mutant in Salmonella Typhimurium and Generation of Complemented Strain}

All primers used in this study are listed in Supplementary Table S1. The $d s r A$ deletion mutant was constructed using the lambda red recombinase system (Datsenko and Wanner, 2000). Knockout mutants were selected for Km resistance and confirmed by colony PCR using specifically designed confirmatory primers.

TABLE 1 | Strains and plasmids used in this study.

\begin{tabular}{|c|c|c|}
\hline & Description & Reference \\
\hline \multicolumn{3}{|l|}{ Strain } \\
\hline WT & $\begin{array}{l}\text { Salmonella enterica serovar } \\
\text { Typhimurium SB300 }\end{array}$ & Pati et al., 2013 \\
\hline$\Delta \mathrm{DsrA}$ & $\begin{array}{l}\text { ADsrA::Km S. Typhimurium } \\
\text { SB300 (Knockout strain) }\end{array}$ & This study \\
\hline$\Delta \operatorname{lnvC}$ & $\begin{array}{l}\text { (invC::aphT; S. Typhimurium } \\
\text { SB300 (Knockout strain) }\end{array}$ & Pati et al., 2013 \\
\hline cDsrA & $\begin{array}{l}\Delta \text { DsrA::pZE12-dsrA S. } \\
\text { Typhimurium SB300 } \\
\text { (Complemented strain) }\end{array}$ & This study \\
\hline \multicolumn{3}{|l|}{ Plasmid } \\
\hline pKD46 & $\begin{array}{l}\lambda \text { red recombinase expressing } \\
\text { plasmid }\left(A m p^{r}\right)\end{array}$ & Datsenko and Wanner, 2000 \\
\hline pKD3 & $\begin{array}{l}\text { Plasmid containing FRT-flanked } \\
\text { chloramphenicol resistance } \\
\text { gene }\end{array}$ & Datsenko and Wanner, 2000 \\
\hline pKD4 & $\begin{array}{l}\text { Plasmid containing FRT-flanked } \\
\text { kanamycin resistance gene }\end{array}$ & Datsenko and Wanner, 2000 \\
\hline pZE12-Iuc & $\begin{array}{l}\text { sRNA complementing vector } \\
\left(A m p^{r}\right)\end{array}$ & Urban and Vogel, 2007 \\
\hline pZE12-dsrA & DsrA expressing vector $\left(A m p^{r}\right)$ & This study \\
\hline
\end{tabular}


The complementation construct of $d s r A$ was based on the pZE12-luc vector (Urban and Vogel, 2007). The $d s r A$ gene was amplified from the genome of S. Typhimurium SB300 (Accession No. FQ312003.1) using primers dsraclF and dsraclR with $\mathrm{n} p f u$ special polymerase (Enzynomics, South Korea). The sense primer anneals to the +1 site of the sRNA gene leaving a $5^{\prime}$-blunt end in the PCR amplicon. The anti-sense primer adds an Xbal site to its $3^{\prime}$ end. Following gel purification, the amplified product was digested with XbaI (New England Biolabs, USA) and subsequently gel extracted. The pZE12-luc vector was amplified with primers pLlacOB and pLlacOC to generate a linear fragment as mentioned above. plLacOC has a $5^{\prime}$ monophosphate required for cloning. Digestion with $\mathrm{XbaI}$ generates a fragment of $\sim 2.2 \mathrm{~Kb}$ that carries the PLlacO promoter (from the position -1), an ampicillin resistance cassette, a ColE1 replicon and a strong $\mathrm{rrnB}$ terminator, followed by the XbaI sticky end. XbaI digested products were ligated for $1 \mathrm{~h}$ at $22^{\circ} \mathrm{C}$ to yield the ligated construct pZE12-dsrA. The orientation of the insert was confirmed using the primer set dsraclF and pLlacOC. The construct was subsequently transformed into the isogenic $d s r A$ mutant $(\triangle \mathrm{DsrA})$ and selected on Amp plates to yield the complemented strain (cDsrA).

\section{Quantitative Reverse Transcription PCR Assays}

Salmonella Typhimurium RNA was extracted at pH 7.5 (prior to adaptation), $\mathrm{pH} 4.4$ (1 $\mathrm{h}$ post adaptation) and $\mathrm{pH} 3.1$ (1 h post challenge) from ATR cultures. Following RNase free DNase1 (Thermo Fisher Scientific, USA) treatment, cDNA was synthesized using Hi-cDNA Synthesis Kit (HIMEDIA, India). Quantitative reverse transcription polymerase chain reaction (qRT-PCR) was performed in triplicate using Kapa Sybr Fast qPCR Master Mix (2x; Kapa Biosystems, USA) with appropriately diluted cDNA templates. The guanylate monophosphate kinase gene $(g m k)$ was used as an internal control after ensuring its suitability under acid stress.

For Salmonella pathogenicity island-1 (SPI-1) and flagellin gene expression, $\triangle \mathrm{DsrA}$ and $S$. Typhimurium SB300 (WT) were grown in Luria-Bertani (LB) broth $(0.3 \mathrm{M} \mathrm{NaCl})$ and subcultured for $4 \mathrm{~h}\left(37^{\circ} \mathrm{C}, 120 \mathrm{rpm}\right)$. Subsequent RNA extraction, purification, cDNA synthesis and qRT-PCR were performed as mentioned above. All assays were performed with three biological replicates in triplicate.

\section{Motility Assays}

Motility assays were performed in triplicate on soft agar plates $(0.3 \% \mathrm{w} / \mathrm{v}$ agar $)$ prepared in LB. Briefly, $1 \mu \mathrm{l}$ of bacterial culture was spotted at the center of each plate, incubated for $8 \mathrm{~h}$ at $37^{\circ} \mathrm{C}$ and the diameter of motile cell growth was measured.

\section{Adhesion and Invasion Assays}

Adhesion and invasion assays were performed in HCT116 and HeLa colonic cell lines as described previously (Pati et al., 2013). In brief, colon epithelial cells were grown in Dulbecco's modified Eagle's medium (DMEM; Invitrogen, USA) supplemented with $10 \%$ fetal bovine serum (FBS) in 24 -well plates at $37^{\circ} \mathrm{C}$ in a $\mathrm{CO}_{2}$ incubator until $80 \%$ confluent. Cells were washed with phosphate buffered saline (PBS) twice, followed by the addition of $500 \mu \mathrm{l}$ supplemented DMEM (without antibiotic) prior to infection. Bacterial cultures were grown overnight in LB broth $(0.3 \mathrm{M} \mathrm{NaCl})$ at $37^{\circ} \mathrm{C}$ and $120 \mathrm{rpm}$ with sub-culturing (1:20 dilution) into fresh medium until an $\mathrm{OD}_{600}$ of 0.6 . Adhesion studies were performed by placing both 24-well plates and inoculum on ice for $15 \mathrm{~min}$ before infection. Infection was carried out at a multiplicity of infection (MOI) of 10 with subsequent incubation for half an hour on ice. Invasion assays were performed with a MOI of 10 , followed by a $50 \mathrm{~min}$ incubation at $37^{\circ} \mathrm{C}$ and $5 \% \mathrm{CO}_{2}$. Cells were subsequently washed twice with PBS and $500 \mu l$ gentamicin (100 $\mu \mathrm{g} / \mathrm{ml})$ containing DMEM was added and incubated for 2 h. Following incubation, cells were twice washed in PBS, lysed with $500 \mu \mathrm{l}$ lysis solution (PBS containing $0.1 \%$ sodium desoxycholate) and suitable dilution plated on LB agar plates with appropriate antibiotics. Adhesion and invasion were calculated as percentages of the number of bacteria recovered, from the total bacteria inoculated. All assays were performed with three biological replicates in triplicate.

\section{Mice Infection and Histopathological Evaluation}

This study utilized C57BL/6 mice that were maintained at the animal house of School of Biotechnology, KIIT University, Odisha, India. Six-eight weeks old Sm pre-treated mice were used in this study, as previously described (Pati et al., 2013). WT, $\Delta$ DsrA, and cDsrA were grown overnight in LB broth and subsequently sub-cultured (1:20) for $4 \mathrm{~h}$. Mice were infected in groups of 5 (oral gavage) with $\sim 10^{7}$ CFU. Bacterial loads in the cecum content, mesenteric lymph node (MLN), spleen and liver were determined $72 \mathrm{~h}$ p.i. by plating suitable dilutions of homogenates on MacConkey agar plates supplemented with the appropriate antibiotics. Histopathological evaluation was performed as previously described (Pati et al., 2013). Briefly, Stained cryosections of the cecum were scored in a blinded manner by two pathologists on the basis of changes indicating sub-mucosal oedema, polymorphonuclear neutrophil (PMN) infiltration, goblet cell loss and epithelial ulceration (Barthel et al., 2003). Pathological scores ranged from 0 to 13 arbitrary units corresponding to levels of inflammation including intact intestines showing no inflammation (pathoscore 0); very mild inflammation (pathoscore 1-2); slight inflammation (pathoscore 3-4); intermediate inflammation (5-8); and significant inflammation (9-13).

\section{Statistical Analysis}

All data represents triplicate experiments with mean \pm standard deviation. Two-way Analysis of Variance (ANOVA) and $t$-tests were used to analyze significant differences. GraphPad Prism version 6.0 was used for all analyses.

\section{Ethical Statement}

All in vivo experiments were performed according to the guidelines of the Institutional Animal Ethics Committee 
(IAEC), KIIT University bearing the approval number: KSBT/IAEC/2015/MEET-1/A2.

\section{RESULTS}

\section{S. Typhimurium ATR}

Prior to determining the expression profile of $d s r A$ under the ATR, WT was tested for its ability to mount an effective ATR in MEM. Both adapted (mid log phase cells grown for $60 \mathrm{~min}$ at pH4.4) and un-adapted (mid log phase) cells were tested for their ability to survive severe $\mathrm{pH}$ exposure $(\mathrm{pH} 3.1 \pm 0.1)$ at various time points post acid challenge. As expected, adapted cells with their enhanced ability to survive harsh $\mathrm{pH}$, displayed greater survival at all time points post challenge in contrast to normal un-adapted cells (Figure 1A). This result is in line with previously reported data in the literature (Bourret et al., 2008).

\section{S. Typhimurium dsrA Expression Is Up-regulated under the ATR}

The 87 bp sRNA DsrA has been reported to be a regulator of acid resistance in E. coli (Lease et al., 2004) and shares about $83 \%$ similarity at the nucleotide level with that of $S$. Typhimurium (Supplementary Figure S1). To determine the expression of $d s r A$ under the ATR, qRT-PCR analysis revealed $d s r A$ to be upregulated two and threefold under acid adaptation and challenge, respectively, relative to levels at pH7.5 (Figure 1B). Guanylate monophosphate kinase $(g m k)$ was used as the reference gene after ensuring its levels did not fluctuate under the observed conditions.

\section{Role of DsrA in the ATR of \\ S. Typhimurium}

To study the influence of DsrA on the ability of $S$. Typhimurium to induce an effective ATR, we compared the survival displayed by adapted cultures of $\triangle \mathrm{DsrA}, \mathrm{WT}$ and $\mathrm{cDsrA}$ to acid challenge as mentioned above after ensuring the growth of both $\triangle \mathrm{DsrA}$ and cDsrA was not hampered (Figure 1C, inset). Interestingly, $\triangle$ DsrA was able to mount an ATR, however, there was a twofold decrease in viable cells at all time points post challenge, relative to both WT and cDsrA (Figure 1C).

\section{Motility Assays and Expression Analysis of Flagellin Structural Genes fliC and fljB}

Motility assays of $\triangle \mathrm{DsrA}$, WT control and cDsrA indicated reduced motility in the mutant while the cDsrA remained comparable to the WT (Figure 2A). To further investigate this reduction in motility, we determined the relative expression of flagellin structural components $f l i C$ and $f l j B$. qRT-PCR analysis revealed $f l i C$ and $f l j B$ to be down regulated 4 and 12 -fold, respectively, in $\triangle \mathrm{DsrA}$ relative to the WT strain, under SPI-1 inducing conditions (Figure 2A inset). The reduced expression of these genes accounts for the observed motility phenotype of $\Delta$ DsrA.

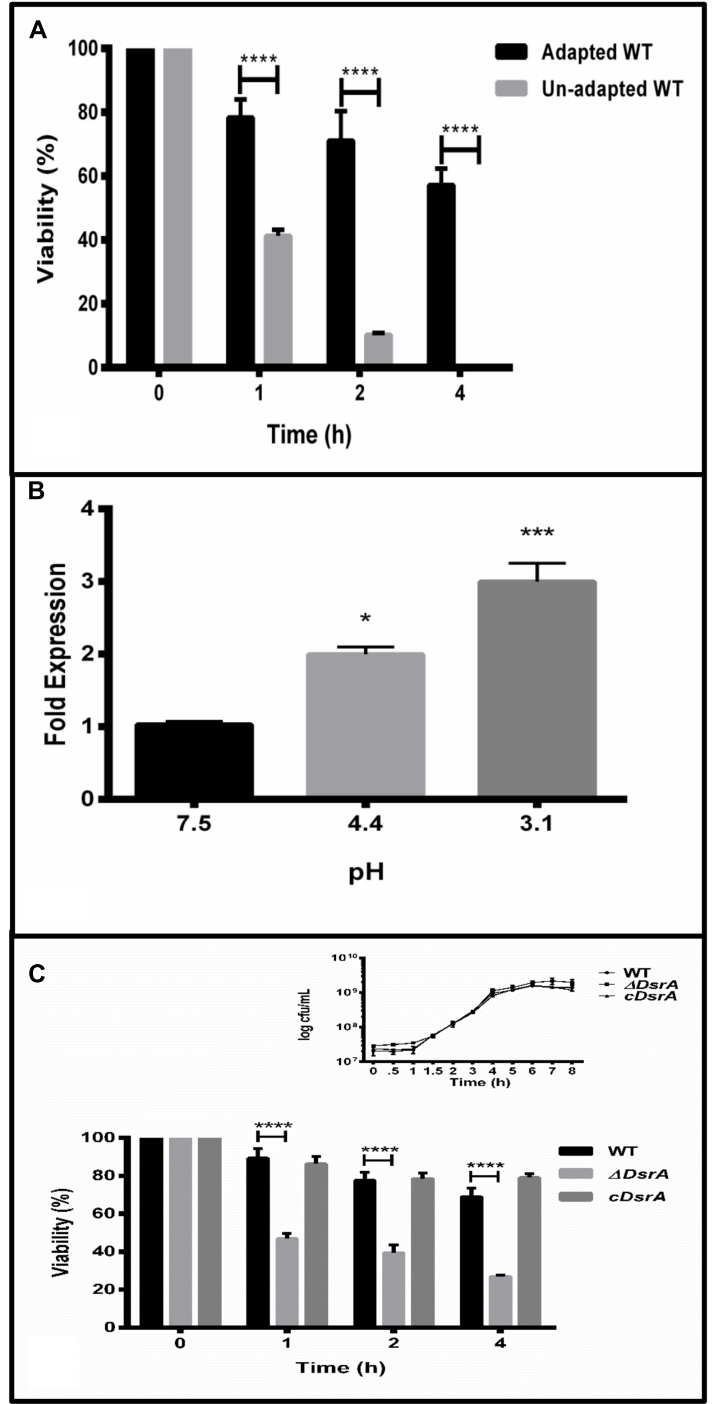

FIGURE 1 | (A) The log-phase acid tolerance response (ATR) of Salmonella Typhimurium. Overnight grown cultures were sub-cultured into minimal EG medium (MEM) at a pH of 7.5 till $\mathrm{OD}_{600}$ of 0.4 . Cultures to be adapted were adjusted to $\mathrm{pH} 4.4 \pm 0.1$ ( $3 \mathrm{~N} \mathrm{HCl}$ ) for $1 \mathrm{~h}$. Un-adapted cultures were left untouched. All flasks were subsequently challenged by adjusting $\mathrm{pH}$ to $3.1 \pm 0.1(3 \mathrm{~N} \mathrm{HCl})$ and grown for 1,2 , and $4 \mathrm{~h}$ post challenge. Viable cells were determined by plating of appropriate dilutions on selective plates. (B) Quantitative reverse transcription polymerase chain reaction (GRT-PCR) of dsrA under normal $(\mathrm{pH} \mathrm{7.5)}$, adaptation $(\mathrm{pH} 4.4)$ and challenge $(\mathrm{pH} 3.1,1 \mathrm{~h})$, respectively. gmk (guanylate monophosphate kinase) was used as the internal control after verifying its suitability under the ATR. (C) ATR of WT, $\triangle \mathrm{DsrA}$ and complemented strain (cDsrA). All experiments were performed in triplicate with data represented as mean \pm SD. Statistical significance: ${ }^{*} P<0.05$, *** $P<0.001,{ }^{* * * *} P<0.0001$

\section{$\Delta$ DsrA Displays Mildly Attenuated Adhesion, while Invasion Is Highly Deficient In Vitro}

To study the role of DsrA in adhesion to epithelial cells, both HeLa (cervical cancer line) and HCT116 (colon cancer line) cells 

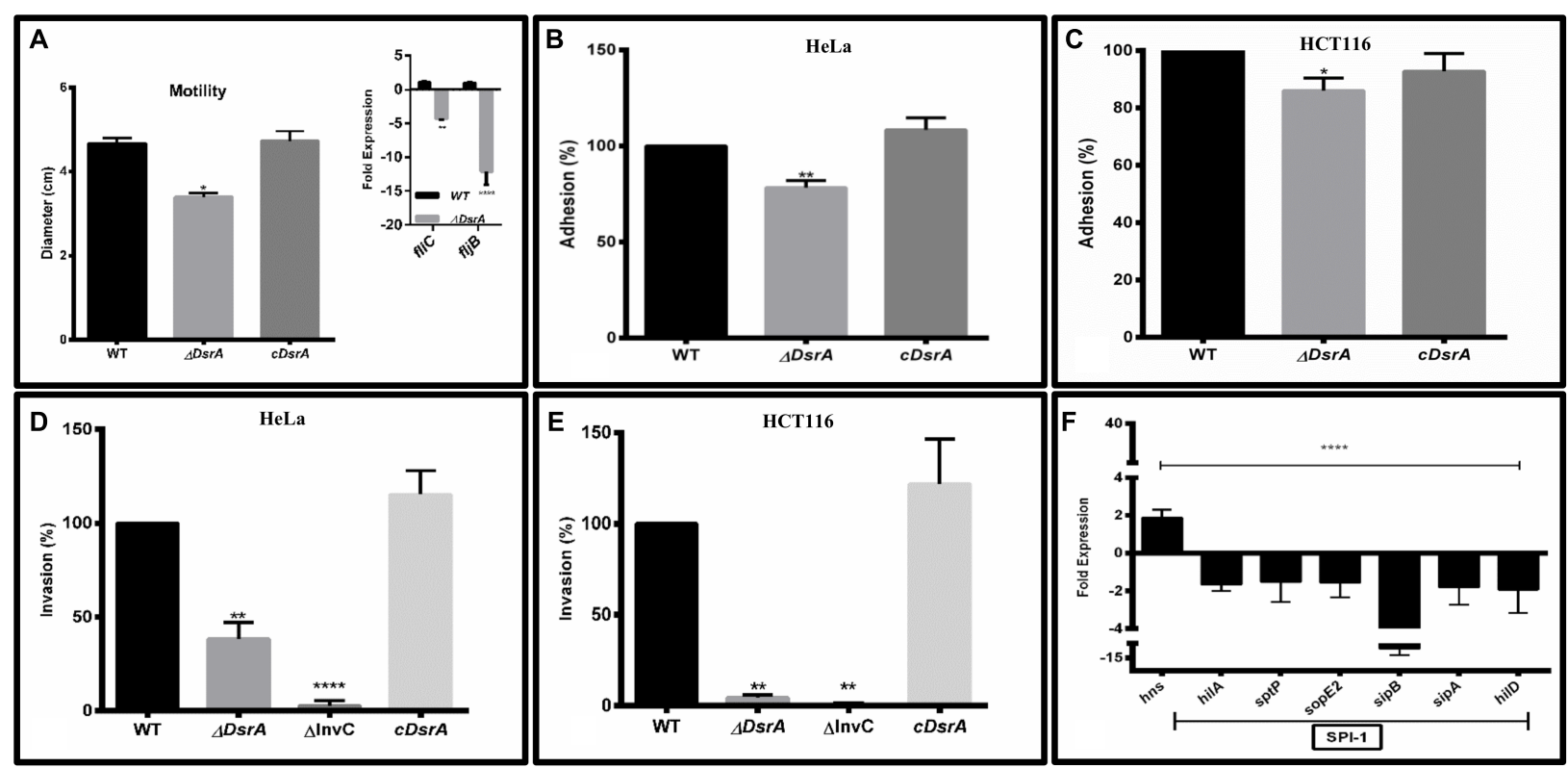

FIGURE 2 | In vitro characterization of $\Delta$ DsrA. (A) Motility assay of isogenic dsrA mutant $\triangle \mathrm{DsrA}$, cDsrA, and WT S. Typhimurium (SB300). qRT-PCR analysis of the flagellin structural genes fliC and fljB are shown in the inset. Guanylate monophosphate kinase ( $g m k$ ) was used as an internal control. (B-E) Adhesion and invasion assays of $\triangle \mathrm{DsrA}$, invasion deficient negative control $\Delta \mathrm{InvC}$, cDsrA, and WT in HeLa and HCT116 cell lines. (F) qRT-PCR analysis of $h n s$ and Salmonella pathogenicity island 1 (SPI-1) associated genes in WT and $\triangle$ DsrA. gmk served as an internal control. All experiments were performed in triplicate with data represented as mean $\pm \mathrm{SD}$. Statistical significance: ${ }^{*} P<0.05, * * P<0.01,{ }^{* * * *} P<0.0001$.

were inoculated with $\triangle \mathrm{DsrA}, \mathrm{cDsrA}$, and WT control. The $d s r A$ mutant displayed mild attenuation in adhesion of about 86 and 78\% in HCT116 and HeLa, respectively, relative to both WT and $\mathrm{cDsrA}$ which displayed comparable adhesion percentages (Figures 2B,C). We hypothesize this mild reduction may be due to an insufficiency in a number of factors including various fimbrae (Type 1, Curli, Pef, and Std) and pathogenicity island associated genes (sipC, sipD, siiE, and $\operatorname{sipB}$, etc.) (Fàbrega and Vila, 2013).

To determine the role of DsrA in the context of bacterial invasion, both HCT116 and HeLa cells were inoculated with $\triangle \mathrm{DsrA}$ and the invasion efficiency was compared to $\triangle \mathrm{InvC}$ (invasion deficient negative control), cDsrA and WT control. $\triangle$ DsrA exhibited a significant lower invasion phenotype of approximately 4.5 and 38\% in HCT116 and HeLa, respectively, relative to both WT and cDsrA (Figures $2 \mathrm{D}, \mathrm{E}$ ). The reduced invasion of the mutant strain may be attributed to a deficiency in the functioning of type 3 secretion system-1 (T3SS-1) which is necessary for bacterial invasion (Pati et al., 2013).

\section{Deletion of dsrA Altered the Expression Profile of Several Genes Associated with Invasion}

To determine the role of DsrA in regulating SPI-1, we analyzed the expression of DsrA target hns along with a panel of SPI1 master regulators and effectors in the $\triangle \mathrm{DsrA}$ strain relative to the WT, under SPI-1 inducing conditions, using qRT-PCR (Figure 2F). This is based on the observation that $\mathrm{H}-\mathrm{NS}$ has been shown to repress a number of pathogenicity islands in Salmonella (Fàbrega and Vila, 2013). The expression of hns was found to be up-regulated 1.8-fold in the mutant while the SPI-1 master regulator hilA was down-regulated 1.6-fold. Additionally, several effectors namely $s p t P, \operatorname{sop} E 2, \operatorname{sip} A$, and $\operatorname{Sip} B$ were repressed along with another regulator hilD in $\triangle \mathrm{Dsr} A$, thus further enforcing the reduced invasive phenotype.

\section{$\Delta$ DsrA Displayed Competitive Colonization But Was Unable to Cause Colitis in a Sm Pre-treated Mouse Model}

We compared the virulence profile of $\triangle \mathrm{DsrA}$ to that of cDsrA and WT in C57BL/6 mice. In all cases, similar levels of colonization were observed with $\triangle \mathrm{DsrA}$ displaying a mild decrease in MLN colonization (Figure 3A). Of note, $\triangle \mathrm{DsrA}$ was unable to cause colitis displaying no inflammation, edema or loss of goblet cells $72 \mathrm{hpi}$. This is in stark contrast to that observed in the case of cDsrA and WT, both of which displayed severe inflammation, oedema, and loss of goblet cells at the same time interval (Figures 3B-D). Pathological scoring revealed cecum infected with $\Delta$ DsrA to have a pathoscore of 2.3 while that infected with WT and cDsrA was 11.5 and 9.8, respectively (Figure 3).

\section{DISCUSSION}

The ability of Salmonella to sense and adapt to changes in its environment is essential to its survival both in nature and within 


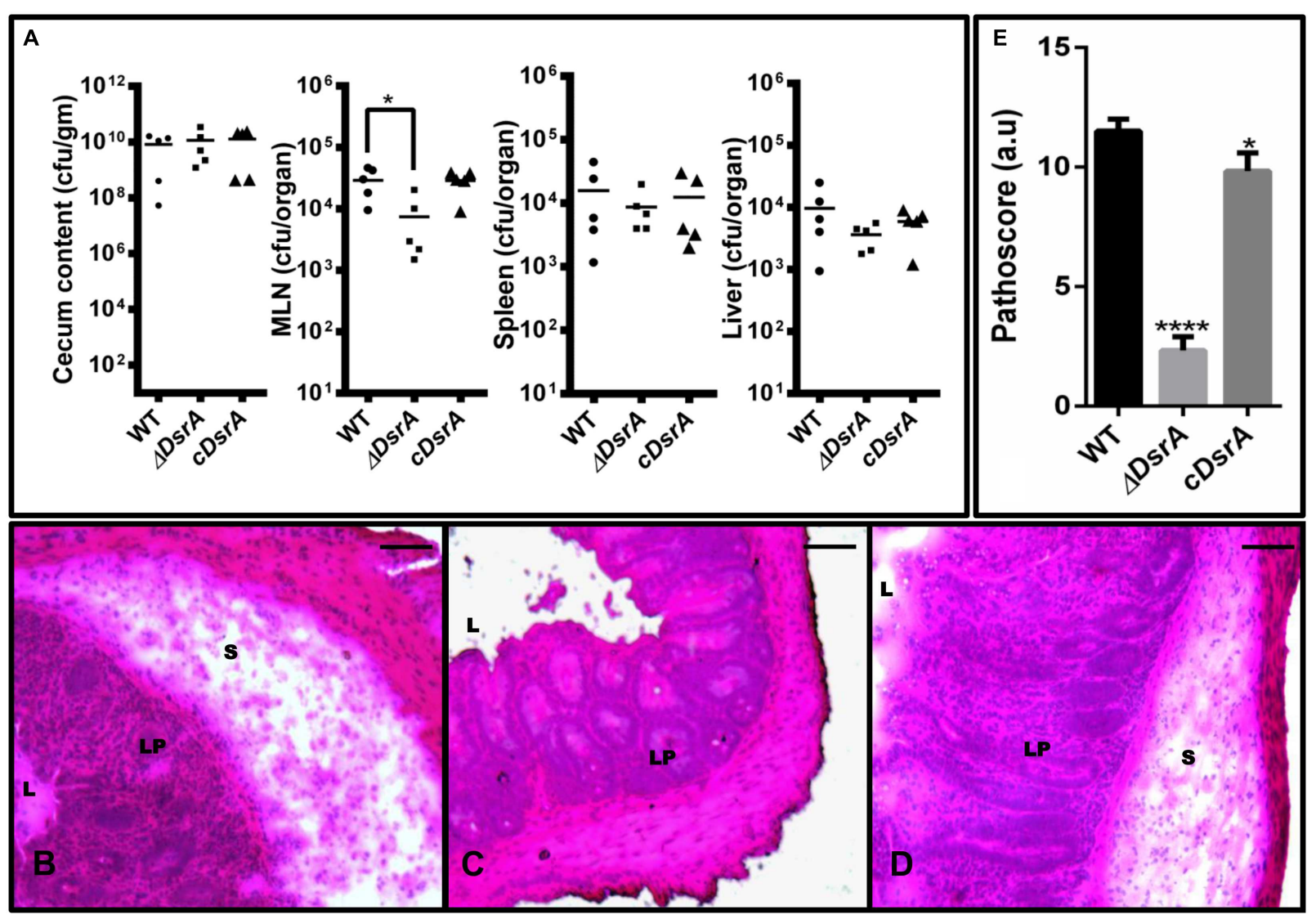

FIGURE 3 | Colonization and cecal inflammation by WT, $\triangle$ DsrA, and cDsrA in C57BL/6 mice. (A) Streptomycin-pretreated mice were infected with the above strains, sacrificed $72 \mathrm{~h}$ p.i. and bacterial loads in the cecum, messenteric lymph node (MLN), liver and spleen were determined. (B-D) Histopathological assessment of the cecal tissue from animals infected with WT, $\triangle \mathrm{Dsr} A$ and $\mathrm{cDsrA}$, respectively. (E) Analysis of cecal pathology by a semi-quantitative method as mentioned in the materials and methods. Bars, $200 \mu \mathrm{m}$. S, submucosa; L, lumen; Lp, lamina propria. Statistical significance: ${ }^{*} P<0.05,{ }^{* * * * *} P<0.0001$.

its host. One such stress that Salmonella must overcome is that of acid. The system that enables this is termed the ATR and it allows Salmonella to survive in normally lethal acidic pH levels following adaptation to mild acidic conditions (Foster, 1993; Garcia-del Portillo et al., 1993; Lin et al., 1995). This adaptation process involves the production of numerous ASPs and the upregulation of systems that help in the maintenance of intracellular $\mathrm{pH}$ and consumption of excess protons within the bacterium (Audia et al., 2001; Ryan et al., 2015). Additionally, successful pathogenesis requires a bacterium to regulate certain host cell functions so as to be able to establish an infection. In Salmonella, genes belonging to SPIs play an important role in invasion of epithelial cells and intracellular survival. In addition to the above mentioned genes, sRNAs have recently been implicated in a number of stress and virulence pathways. Transcriptomics and genomics based approaches have predicted the existence of a number of sRNAs under various conditions, however, the vast majority remain functionally uncharacterized and thus, a complete strata of regulation seems to be lacking (Erdmann et al., 2001). One such sRNA, DsrA has been shown to regulate resistance to acid stress in E. coli and be repressed within intracellular Salmonellae within fibroblasts. (Lease and Belfort,
2000; Lease et al., 2004; Ortega et al., 2012). To this effect, we speculated a role for DsrA in the ATR of $S$. Typhimurium and characterized its effect on virulence.

The $d s r A$ gene is induced under the $S$. Typhimurium ATR in MEM with maximum induction at $\mathrm{pH}$ 3.1. Further, deletion of $d s r A$ was found to reduce the effectiveness of the ATR with lower percent viability at all three time points post acid challenge compared to wild-type SB300. This observation suggests a role for DsrA in the ATR of Salmonella, possibly through regulation of the stress response sigma factor rpoS (Lease et al., 2004), a first for any sRNA, although further work needs to be performed to confirm this hypothesis The link between acid survival and pathogenesis is an important characteristic for enteric pathogens such as Salmonella, which are constantly exposed to low $\mathrm{pH}$ during their course of infection in a host as well as in their habitat (Suar and Ryan, 2015). To this effect, we have further characterized the role of DsrA in the virulence of this pathogen.

$\Delta$ DsrA was found to exhibit slightly reduced motility and displayed a mild reduction in its ability to adhere to both HCT and HeLa intestinal epithelial cell lines. Intriguingly, the mutant displayed a significantly reduced ability to invade the 
aforementioned cell lines. The nucleoid protein and global silencer of transcription hns, has been shown to be repressed at the translational level by DsrA in E. coli although little is known as to whether this occurs by the enhancement of mRNA turnover or by the occlusion of ribosomes (Lease et al., 1998). H-NS in turn is a silencer of the SPI-1 master regulator hilA (Fàbrega and Vila, 2013) which is responsible for the induction of various effectors which are required for successful invasion of epithelial cells (Hapfelmeier et al., 2004). Thus, we suggest the sRNA DsrA mediates its effects on SPI-1 through this regulatory circuit which accounts for the highly reduced invasive phenotype. To validate this, we determined the expression levels of $h n s$ and several SPI1 associated genes in $\triangle$ DsrA relative to wild-type under SPI-1 inducing conditions. Interestingly, we found $h n s$ levels to increase in a subtle but reproducible manner in accordance with what has already been reported (Lease et al., 1998), while hilA and several effector levels were repressed. The decrease in expression has a direct consequence of inhibiting invasion of epithelial cells.

Our in vivo data revealed $\triangle$ DsrA colonization of the cecum and other systemic organs to be comparable to that of wild-type SB300 at $72 \mathrm{hpi}$, however, there was considerable difference in the observed cecal pathology. $\triangle \mathrm{D} s \mathrm{~A}$ A was found to be attenuated in developing gut inflammation at $72 \mathrm{hpi}$. This attenuation cannot be explained by a mere reduction in the expression of SPI-1 genes as earlier studies have shown Salmonella SPI-1 mutants to induce comparable cecal inflammation to the WT at the same time point (Hapfelmeier et al., 2005). On the other hand, several studies on Salmonella as a model for pathogenesis have revealed flagellin to play a major role in triggering proinflammatory responses in epithelial cells (Lyons et al., 2004). Double mutants of the flagellin structural genes $f l i C$ and $f l j B$ were found to be unable to induce a proinflammatory response (Zeng et al., 2003). Consequently, our expression analysis revealed significantly lower levels of expression of these two genes in $\triangle \mathrm{DsrA}$, suggesting a possible cause for the observed phenotype in addition to the reduced motility observed in the $d s r A$ mutant.

To conclude, the enteric pathogen Salmonella must efficiently sense and respond to various environmental challenges, acidic $\mathrm{pH}$ being of prime importance. To achieve this, the bacterium is

\section{REFERENCES}

Allam, U. S., Krishna, M. G., Sen, M., Thomas, R., Lahiri, A., Gnanadhas, D. P., et al. (2012). Acidic pH induced STM1485 gene is essential for intracellular replication of Salmonella. Virulence 3, 112-125. doi: 10.4161/viru.19029

Altuvia, S. (2007). Identification of bacterial small non-coding RNAs: experimental approaches. Curr. Opin. Microbiol. 10, 257-261. doi: 10.1016/j.mib.2007.05.003

Audia, J. P., Webb, C. C., and Foster, J. W. (2001). Breaking through the acid barrier: an orchestrated response to proton stress by enteric bacteria. Int. J. Med. Microbiol. 291, 97-106. doi: 10.1078/1438-4221-00106

Baik, H. S., Bearson, S., Dunbar, S., and Foster, J. W. (1996). The acid tolerance response of Salmonella typhimurium provides protection against organic acids. Microbiology 142, 3195-3200. doi: 10.1099/13500872-142-11-3195

Bang, I. E. L. S. O. O., Kim, B. A. E. H., Foster, J. W., and Park, Y. K. (2000). OmpR regulates the stationary-phase acid tolerance response of Salmonella enterica serovar typhimurium. J. Bacteriol. 182, 2245-2252. doi: 10.1128/JB.182.8.22452252.2000

Barthel, M., Hapfelmeier, S., Quintanilla-Martínez, L., Kremer, M., Rohde, M., Hogardt, M., et al. (2003). Pretreatment of mice with streptomycin provides able to detect low $\mathrm{pH}$ through various two component systems namely PhoP/Q, EnvZ/OmpR, etc., followed by the initiation of a specific response termed the ATR (Bearson et al., 1998; Bang et al., 2000; Ryan et al., 2015). Furthermore, survival and adaptation to acidic $\mathrm{pH}$ is essential to the virulence of this pathogen. Our study has highlighted the role of the sRNA DsrA in the ATR of Salmonella. Additionally, we have shown DsrA to be required for the invasion of epithelial cell lines possibly through the hns, hilA, SPI-1 regulatory route. Virulence studies in mice demonstrated DsrA may be required for eliciting an inflammatory response mediated through possible regulatory effects on the flagellin structural genes $f l i C$ and $f l j B$. However, it remains necessary to clarify the effect of DsrA on these genes. Successful Salmonella pathogenesis requires that the bacterium survive a plethora of stressors in its environment, a major one being acidic $\mathrm{pH}$ of the stomach and SCV. Additionally, there must be a coordinated regulation of various pathogenicity island associated genes to ensure successful invasion and survival within host cells (Fàbrega and Vila, 2013). Consequently, this work has served to highlight the importance of the link between acid survival and virulence with another strata of regulation namely, sRNAs being brought into the fold. Finally, the phenotype of non-invasiveness of $\triangle \mathrm{DsrA}$ could be exploited for the development of SPI-1 attenuated strains without disrupting SPI-1 genes.

\section{AUTHOR CONTRIBUTIONS}

DR and MS conceived and designed the experiments. DR, UO, SJ, and CP performed the experiments. DR and MS analyzed and wrote the paper.

\section{SUPPLEMENTARY MATERIAL}

The Supplementary Material for this article can be found online at: http://journal.frontiersin.org/article/10.3389/fmicb. 2016.00599

a Salmonella enterica serovar Typhimurium colitis model that allows analysis of both pathogen and host. Infect. Immun. 71, 2839-2858. doi: 10.1128/IAI.71.5.2839

Bearson, B. L., Wilson, L., and Foster, J. W. (1998). A low pH-inducible, PhoPQdependent acid tolerance response protects Salmonella typhimurium against inorganic acid stress. J. Bacteriol. 180, 2409-2417.

Bourret, T. J., Porwollik, S., McClelland, M., Zhao, R., Greco, T., Ischiropoulos, H., et al. (2008). Nitric oxide antagonizes the acid tolerance response that protects Salmonella against innate gastric defenses. PLOS ONE 3:e1833. doi: 10.1371/journal.pone.0001833

Datsenko, K. A., and Wanner, B. L. (2000). One-step inactivation of chromosomal genes in Escherichia coli K-12 using PCR products. Proc. Natl. Acad. Sci. U.S.A. 97, 6640-6645.

Erdmann, V. A., Barciszewska, M. Z., Szymanski, M., Hochberg, A., de Groot, N., Barciszewski, J., et al. (2001). The non-coding RNAs as riboregulators. Nucleic Acids Res. 29, 189-193. doi: 10.1093/nar/29.1.189

Fàbrega, A., and Vila, J. (2013). Salmonella enterica serovar Typhimurium skills to succeed in the host: virulence and regulation. Clin. Microbiol. Rev. 26, 308-341. doi: 10.1128/CMR.00066-12 
Foster, J. (1993). The acid tolerance response of Salmonella typhimurium involves transient synthesis of key acid shock proteins. J. Bacteriol. 175, 1981-1987.

Foster, J. W., and Hall, H. K. (1990). Adaptive acidification tolerance response of Salmonella typhimurium. J. Bacteriol. 172, 771-778.

Galán, J. E. (2001). Salmonella interactions with host cells: type III secretion at work. Annu. Rev. Cell Dev. Biol. 17, 53-86. doi: 10.1146/annurev.cellbio.17.1.53

Garcia-del Portillo, F., Foster, J. W., and Finlay, B. B. (1993). Role of acid tolerance response genes in Salmonella typhimurium virulence. Infect. Immun. $61,4489-4492$.

Gong, H., Vu, G.-P., Bai, Y., Chan, E., Wu, R., Yang, E., et al. (2011). A Salmonella small non-coding RNA facilitates bacterial invasion and intracellular replication by modulating the expression of virulence factors. PLoS Pathog. 7:e1002120. doi: 10.1371/journal.ppat.1002120

Hapfelmeier, S., Ehrbar, K., Stecher, B., Kremer, M., Hardt, W., and Barthel, M. (2004). Role of the Salmonella pathogenicity island 1 effector proteins SipA, SopB, SopE, and SopE2 in Salmonella enterica subspecies 1 serovar typhimurium colitis in streptomycin-pretreated mice. Infect. Immun. 72, 795-809. doi: 10.1128/IAI.72.2.795

Hapfelmeier, S., Stecher, B., Barthel, M., Kremer, M., Muller, A. J., Heikenwalder, M., et al. (2005). The Salmonella pathogenicity island (SPI)-2 and SPI-1 type III secretion systems allow Salmonella serovar typhimurium to trigger colitis via MyD88-dependent and MyD88-independent mechanisms. J. Immunol. 174, 1675-1685. doi: 10.4049/jimmunol.174.3.1675

Hébrard, M., Kröger, C., Srikumar, S., Colgan, A., Händler, K., and Hinton, J. C. (2012). sRNAs and the virulence of Salmonella enterica serovar Typhimurium. RNA Biol. 9, 437-445. doi: 10.4161/rna.20480

Lease, R. A., and Belfort, M. (2000). A trans-acting RNA as a control switch in Escherichia coli: DsrA modulates function by forming alternative structures. Proc. Natl. Acad. Sci. U.S.A 97, 9919-9924. doi: 10.1073/pnas.170281497

Lease, R. A., Cusick, M. E., and Belfort, M. (1998). Riboregulation in Escherichia coli: DsrA RNA acts by RNA: RNA interactions at multiple loci. Proc. Natl. Acad. Sci. U.S.A. 95, 12456-12461. doi: 10.1073/pnas.95.21.12456

Lease, R. A., Smith, D., Mcdonough, K., and Belfort, M. (2004). The small noncoding DsrA RNA is an acid resistance regulator in Escherichia coli $\dagger$. J. Bacteriol. 186, 6179-6185. doi: 10.1128/JB.186.18.6179-6185.2004

Lin, J., Lee, I. S., Frey, J., Slonczewski, J. L., and Foster, J. W. (1995). Comparative analysis of extreme acid survival in Salmonella typhimurium, Shigella flexneri, and Escherichia coli. J. Bacteriol. 177, 4097-4104.

Lyons, S., Wang, L., Casanova, J. E., Sitaraman, S. V., Merlin, D., and Gewirtz, A. T. (2004). Salmonella typhimurium transcytoses flagellin via an SPI2-mediated vesicular transport pathway. J. Cell Sci. 117, 5771-5780. doi: 10.1242/jcs. 01500

Nadim, M., Christofer, C., Darren, S., Tom, E., and Gottesman, S. (1998). DsrA RNA regulates translation of RpoS message by an anti-antisense mechanism, independent of its action as an antisilencer of transcription. Proc. Natl. Acad. Sci. U.S.A. 95, 12462-12467. doi: 10.1073/pnas.95.21.12462

Ortega, Á. D., Gonzalo-Asensio, J., and García-del Portillo, F. (2012). Dynamics of Salmonella small RNA expression in non-growing bacteria located inside eukaryotic cells. RNA Biol. 9, 469-488. doi: 10.4161/rna.19317

Pati, N. B., Vishwakarma, V., Jaiswal, S., Periaswamy, B., Hardt, W.-D., and Suar, M. (2013). Deletion of invH gene in Salmonella enterica serovar Typhimurium limits the secretion of Sip effector proteins. Microbes Infect. 15, 66-73. doi: 10.1016/j.micinf.2012.10.014

Ryan, D., Pati, N. B., Ojha, U. K., Padhi, C., Ray, S., Jaiswal, S., et al. (2015). Global transcriptome and mutagenic analyses of the acid tolerance response of Salmonella enterica serovar typhimurium. Appl. Environ. Microbiol. 81, 8054-8065. doi: 10.1128/AEM.02172-15

Suar, M., and Ryan, D. (2015). Small RNA in the acid tolerance response of Salmonella and their role in virulence. Virulence 6, 105-106. doi: $10.4161 / 21505594.2014 .988543$

Urban, J. H., and Vogel, J. (2007). Translational control and target recognition by Escherichia coli small RNAs in vivo. Nucleic Acids Res. 35, 1018-1037. doi: 10.1093/nar/gkl1040

Vogel, H. J., and Bonner, D. M. (1956). Acetylornithinase of Escherichia coli: partial purification and some properties. J. Biol. Chem. 218, 97-106.

Zeng, H., Carlson, A. Q., Guo, Y., Yu, Y., Collier-Hyams, L. S., Madara, J. L., et al. (2003). Flagellin is the major proinflammatory determinant of enteropathogenic Salmonella. J. Immunol. 171, 3668-3674. doi: 10.4049/jimmunol.171.7.3668

Conflict of Interest Statement: The authors declare that the research was conducted in the absence of any commercial or financial relationships that could be construed as a potential conflict of interest.

Copyright (๑) 2016 Ryan, Ojha, Jaiswal, Padhi and Suar. This is an open-access article distributed under the terms of the Creative Commons Attribution License (CC BY). The use, distribution or reproduction in other forums is permitted, provided the original author(s) or licensor are credited and that the original publication in this journal is cited, in accordance with accepted academic practice. No use, distribution or reproduction is permitted which does not comply with these terms. 